\title{
Alterações radiológicas presentes no sistema respiratório de pacientes com COVID- 19: Uma revisão sistemática de literatura
}

\author{
Radiological changes present in the respiratory system of patients with COVID-19: A systematic
} literature review

Cambios radiológicos presentes en el sistema respiratorio de pacientes con COVID-19: Una revisión sistemática de la literatura

\section{Resumo}

Objetivo: investigar na literatura internacional os achados radiológicos mais frequentes no sistema respiratório de pacientes infectados pelo Sars-CoV-2. Metodologia: Trata-se de uma Revisão Sistemática de Literatura (RSL), a busca sendo realizada nas bases de dados Pubmed e Cochrane Library, sendo utilizadas estratégias de busca abrangentes dos seguintes termos e seus correlatos baseados no Medical Subject Headings (MeSH): Coronavirus Infection, Respiratory Systems, Imaging, Diagnostic. Foi incluído estudos observacionais publicados a partir de 2020 em qualquer idioma. Resultados: após a triagem, 8 estudos foram detectados, com população total de 1.089 pacientes de 6 países diferentes. Discussão: os pacientes infectados pelo Sars-CoV-2 foram avaliados por duas categorias de exames de imagem: Tomografia Computadorizada e Ultrassonografia Pulmonar, e o comprometimento pulmonar está intimamente relacionado com a gravidade e a presença de fatores de riscos. Conclusão: os resultados evidenciam a importância dos achados radiológicos como ferramenta complementar no diagnóstico e prognóstico da COVID-19, embora não deva substituir os exames laboratoriais na confirmação diagnóstica.

Palavras-chave: COVID-19; Diagnóstico por imagem; Sistema respiratório; Técnicas de diagnóstico do sistema respiratório.

\begin{abstract}
Objective: to investigate the most frequent radiological findings in the international literature in the respiratory system of patients infected with Sars-CoV-2. Methodology: This is a Systematic Literature Review (RSL), a search being performed in the Pubmed and Cochrane Library databases, using a comprehensive search for the following terms and their correlates based on the Medical Subject Headings (MeSH): Coronavirus Infection, Respiratory Systems, Imaging, Diagnostic. Observational studies published from 2020 in any language were included. Results: After screening, 8 studies were included, with a total population of 1,089 patients from 6 different countries. Discussion: patients infected by Sars-CoV-2 were obtained by two categories of imaging tests: Computed Tomography and Pulmonary Ultrasonography, and pulmonary involvement is closely related to the severity and presence of risk factors. Conclusion: the results show the importance of radiological findings as a complementary tool in the diagnosis and prognosis of COVID-19, although they should not replace laboratory tests in confirming the diagnosis.
\end{abstract}

Keywords: COVID-19; Diagnostic imaging; Respiratory system; Diagnostic techniques, respiratory system. 


\begin{abstract}
Resumen
Objetivo: investigar los hallazgos radiológicos más frecuentes en la literatura internacional en el sistema respiratorio de pacientes infectados por Sars-CoV-2. Metodología: Se trata de una revisión sistemática de la literatura (RSL), una búsqueda que se realiza en las bases de datos de Pubmed y Cochrane Library, utilizando una búsqueda integral de los siguientes términos y sus correlatos basados en Medical Subject Headings (MeSH): Coronavirus Infection, Respiratory Systems, Imaging, Diagnostic. Se incluyeron estudios observacionales publicados a partir de 2020 en cualquier idioma. Resultados: después del cribado, se incluyeron 8 estudios, con una población total de 1.089 pacientes de 6 países diferentes. Discusión: los pacientes infectados por Sars-CoV-2 se obtuvieron mediante dos categorías de exámenes de imagen: tomografía computarizada y ecografía pulmonar, y la afectación pulmonar está estrechamente relacionada con la gravedad y presencia de factores de riesgo. Conclusión: los resultados muestran la importancia de los hallazgos radiológicos como herramienta complementaria en el diagnóstico y pronóstico de COVID-19, aunque no deben reemplazar las pruebas de laboratorio en la confirmación del diagnóstico.
\end{abstract}

Palabras clave: COVID-19; Diagnóstico por imagen; Sistema respiratorio; Técnicas de diagnóstico del sistema respiratorio.

\title{
1. Introdução
}

Um surto por uma infecção respiratória ocorreu em Wuhan, província de Hubei, na China, e se espalhou rapidamente em todo o país no fim de 2019. O Centro Chinês para Controle de Doenças e Prevenção (CCDC) identificou um novo betacoronavírus chamado 2019-nCoV, agora oficialmente conhecido como Sars-CoV-2, responsável por provocar uma síndrome respiratória aguda grave, a doença COVID-19, originando a pandemia do novo coronavírus (Gorbalenya et al., 2020).

$\mathrm{O}$ coronavírus competem à ordem Nidovirales e à família Coronaviridae. Tendo como subfamília a Coronavirinae, no qual é composta pelos gêneros Betacoronavirus e Alphacoronavirus e, cujos membros infectam mamíferos. O SARS-CoV-2 é um $\beta$-coronavírus (subgênero Sarbecovírus, Subfamília Orthocoronavirinae), e possui um material genético RNA de sentido positivo não segmentado (Woo et al., 2012; Zhu et al., 2020).

O Sars-CoV-2 possui um período de incubação em torno de 5 dias, o que torna o diagnóstico imprescindível, uma vez que a transmissão da COVID-19 ocorre, também, por pacientes assintomáticos, de acordo com Dias (2020) essa taxa de transmissão pode variar entre 30 e 50\%, visto que as partículas respiratórias são o principal meio de contágio, o que demonstra a necessidade do diagnóstico precoce dos pacientes que ainda não apresentam características clínicas da infecção pelo Sars-CoV2, no intuito de diminuir a transmissão (Lima et al., 2020).

Diversas dificuldades podem surgir durante o enfrentamento de uma pandemia, seja a nível social, organizacional, sanitário e/ou hospitalar. Dessa forma, a pandemia do novo coronavírus tem demonstrado a necessidade de infraestrutura, planejamento, conhecimento e antecipação por parte dos gestores e das equipes de saúde, para enfrentar e agir perante um novo agente infeccioso em escala global. Mediante a isso, além da busca de acesso a vacinação, o diagnóstico e tratamento para os sintomas desta nova pneumonia tem se demonstrado um desafio (Chamas, 2020).

Nesse sentido, o uso de exames de imagem pode complementar e contribuir com o diagnóstico e avaliação dos critérios de gravidade ocasionado pela COVID-19 (Mogami, 2021). Embora a confirmação diagnóstica ocorra através da Reação em Cadeia da Polimerase por Transcrição Reversa (RT-PCR), os exames de imagem tornam-se uma ferramenta valiosa no auxílio diagnóstico, além de ser útil no acompanhamento da evolução e detecção de complicações, principalmente em meio à crise sanitária que dificulta o acesso ao RT-PCR, além da sobrecarga dos laboratórios (Rosa, 2020).

Nesse contexto, o conhecimento é vital para o êxito perante um novo desafio. Dessa forma, a produção científica é fundamental para expor padrões e quais características remetem ao desenvolvimento de tal patologia perante a fisiologia humana, como é o caso da COVID-19, em que estudos e pesquisas com evidências científicas tornam-se imprescindíveis. Desse modo, o objetivo dessa revisão sistemática foi investigar na literatura internacional os achados radiológicos mais frequentes no sistema respiratório de pacientes infectados pelo Sars-CoV-2. 


\section{Metodologia}

\subsection{Tipo de Estudo}

A Revisão Sistemática de Literatura (RSL) foi realizada com dados de estudos observacionais publicados a partir de 2020, seguindo os critérios e recomendações dos relatórios para RSL: O PRISMA tem como objetivo ajudar os pesquisadores a melhorarem o relato de revisões sistemáticas e metanálises (Galvão et al., 2015), o Manual da Cochrane para RSL é o guia oficial que apresenta o detalhamento do processo de construção e manutenção desses estudos (Higgins, Green, 2011).

\subsection{Fonte de Informação e Estratégia de Busca}

Nesse contexto, estudos potenciais foram identificados por meio de uma estratégia sensível baseadas no Medical Subject Headings (MeSH), nas bases de dados: PubMed e Cochrane Library, conforme representado no Quadro 1.

Quadro 1. Estratégia de Busca das bases de dados.

\begin{tabular}{|c|c|}
\hline \multirow{2}{*}{ Estratégia PubMed } & (“"Coronavirus Infection”[Mesh] OR Infection, Coronavirus OR Infections, Coronavirus OR Middle \\
& East Respiratory Syndrome) AND ("Respiratory Systems”[Mesh] OR System, Respiratory OR \\
& Systems, Respiratory OR Respiratory Tract OR Respiratory Tracts OR Tract, Respiratory OR Tracts, \\
& Respiratory) AND ("Imaging, Diagnostic”[Mesh] OR Medical Imaging OR Imaging, Medical)). \\
\hline \multirow{3}{*}{ Estratégia Cochrane Library } & (Coronavirus Infection) OR (Infection, Coronavirus) OR (Infections, Coronavirus) OR (Middle East \\
& Respiratory Syndrome) AND (Respiratory Systems) OR (System, Respiratory) OR (Systems, \\
& Respiratory) OR (Respiratory Tract) OR (Respiratory Tracts) OR (Tract, Respiratory) OR (Tracts, \\
Respiratory) AND (Imaging, Diagnostic) OR (Medical Imaging) OR (Imaging, Medical).
\end{tabular}

Fonte: Autores.

\subsection{Critérios de Elegibilidade}

Foram incluídos estudos observacionais disponíveis na íntegra em todos os idiomas publicados em periódicos a partir de 2020, sendo excluídos artigos de natureza não humana e não observacionais, artigos indisponíveis, duplicados, que não se adaptaram ao objetivo do estudo e que tinham população infantil.

\subsection{Triagem e extração dos dados}

Para triagem dos artigos foi utilizado o software Rayyan QCRI®, sendo possível agilizar a triagem inicial de resumos e títulos através de um processo de semiautomação, bastante utilizado em revisões sistemáticas e de extrema importância uma vez que a síntese de diversos estudos em uma revisão sistemática pode fornecer evidências sobre a eficácia das intervenções (Ouzzani et al., 2016). Além disso, foi utilizado uma planilha no software Microsoft Excel® para extração dos dados dos artigos elegíveis e, por fim, adicionados no gerenciador de referências, software Mendeley®.

\subsection{Avaliação da qualidade}

Para realizar a avaliação da qualidade metodológica dos estudos observacionais incluídos foi utilizado a escala Newcastle-Ottawa, que abrange três domínios no processo de avaliação: seleção, comparabilidade e resultados, utilizando um sistema estelar ( 0 a 9), em que os escores mais altos significam melhor qualidade do estudo. Essa avaliação foi realizada por dois pesquisadores independentes, em caso de incerteza foi consultado um terceiro.

\section{Resultados}

\subsection{Seleção dos estudos}

Após a busca, considerando todos os critérios de elegibilidade e filtros, foram identificados 67 artigos, sendo 65 selecionados na PubMed e 02 na Cochrane. Não houve nenhuma duplicata, assim, foi realizada a triagem de títulos e resumos 
de todos, restando 19 estudos com potencial para RSL, dos quais após leitura integral, apenas 8 estudos foram avaliados para elegibilidade. Conforme descrito na Figura 1, baseada a partir do estudo de Page et al. (2021).

Figura 1. Fluxograma PRISMA.

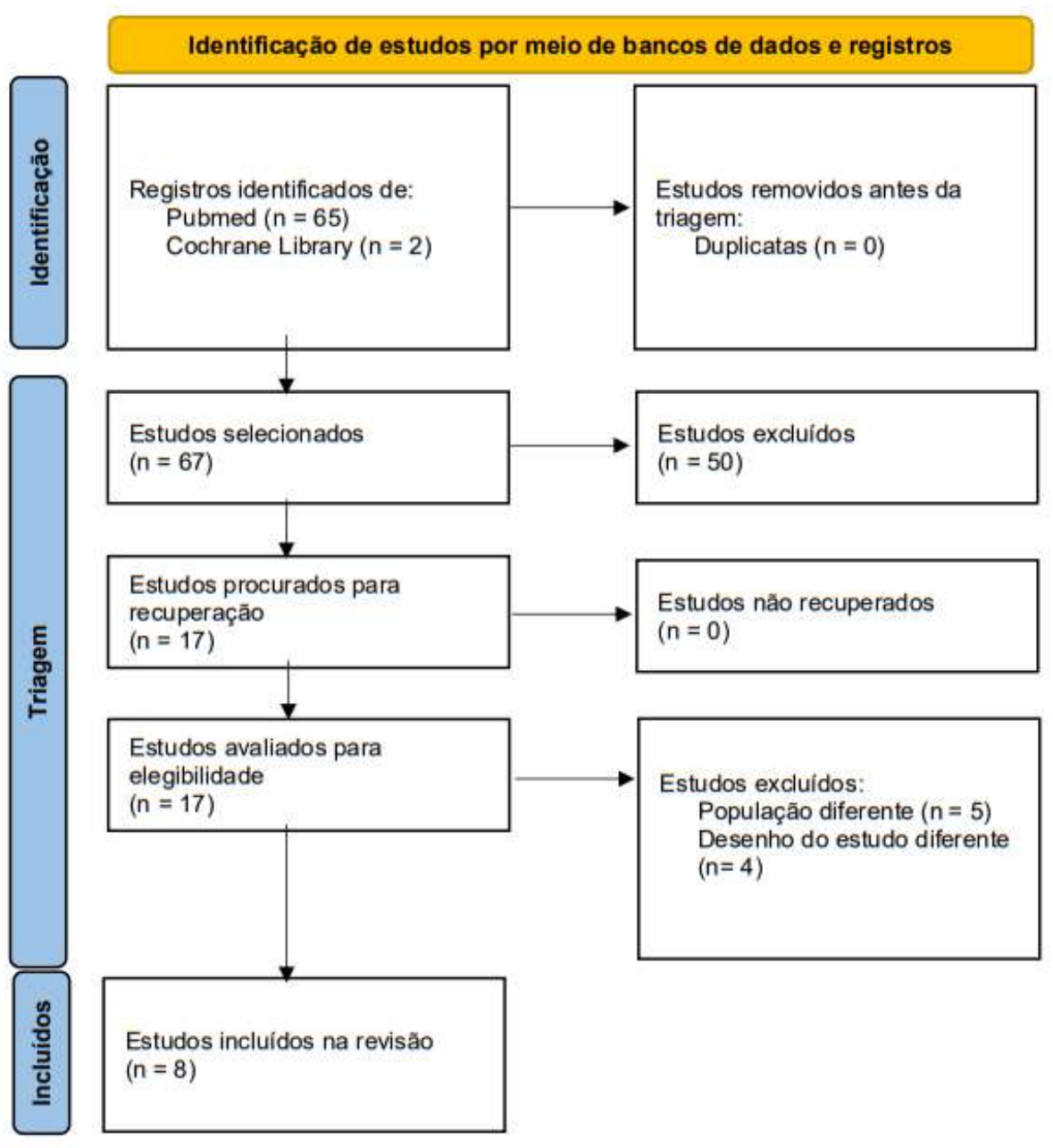

Fonte: Autores.

\subsection{Caracterização dos estudos incluídos}

Esta RSL apresenta estudos de seis países, sendo: (dois) China, (dois) Estados Unidos, (um) Brasil, (um) França, (um) Índia e (um) Itália. Destes, sete estudos foram publicados em 2020 e apenas um em 2021, apresentando uma amostra total de 1.089 pessoas que foram infectadas pelo Sars-CoV-2 confirmadas por RT-PCR e fizeram exame de imagem (tabela 1). 
Tabela 1. Características dos estudos incluídos.

\begin{tabular}{|c|c|c|c|c|}
\hline Título & Autor/ano & $\begin{array}{l}\text { Populaçãa } \\
\text { (n) }\end{array}$ & Intervenção & Desfecho \\
\hline $\begin{array}{c}\text { The pulmonary sequalae in } \\
\text { discharged patients with COVID-19: } \\
\text { a short-term observational study }\end{array}$ & $\begin{array}{l}\text { Liu et al. } \\
(2020)\end{array}$ & 149 & $\mathrm{TC}$ & $\begin{array}{l}\text { Diversas lesões pulmonares surgem em pacientes } \\
\text { com COVID-19, uma parte dessas podem ser } \\
\text { absorvidas completamente sem sequelas. }\end{array}$ \\
\hline $\begin{array}{l}\text { The association of lung ultrasound } \\
\text { images with COVID-19 infection in } \\
\text { an emergency room cohort }\end{array}$ & $\begin{array}{l}\text { Bar et al. } \\
(2020)\end{array}$ & 31 & POCUS & $\begin{array}{c}\text { Devido a identificação de alterações esse modelo } \\
\text { pode facilitar a triagem de pacientes com suspeita } \\
\text { de infecção por COVID - } 19 \text { admitidos na sala de } \\
\text { emergência }\end{array}$ \\
\hline $\begin{array}{l}\text { Temporal changes of CT findings } \\
\text { between non-severe and severe cases } \\
\text { of COVID-19 pneumonia: a multi- } \\
\text { center, retrospective, longitudinal } \\
\text { Study }\end{array}$ & $\begin{array}{l}\text { Dai et al. } \\
(2020)\end{array}$ & 73 & $\mathrm{TC}$ & $\begin{array}{l}\text { As manifestações de TC seguem certos padrões } \\
\text { em diferentes momentos do curso da doença, que } \\
\text { está relacionado à gravidade, progressão e } \\
\text { recuperação da doença }\end{array}$ \\
\hline $\begin{array}{c}\text { Point-of-Care Lung Ultrasound } \\
\text { Findings in Patients with COVID-19 } \\
\text { Pneumonia }\end{array}$ & $\begin{array}{l}\text { Yasukawa } \\
\text { et al. }(2020)\end{array}$ & 10 & POCUS & $\begin{array}{l}\text { Há o surgimento de alterações típicas e a } \\
\text { presença desses achados é útil na avaliação de } \\
\text { pacientes com suspeita de COVID-19. }\end{array}$ \\
\hline $\begin{array}{l}\text { The role of a chest computed } \\
\text { tomography severity score in } \\
\text { coronavirus disease } 2019 \text { pneumonia }\end{array}$ & $\begin{array}{l}\text { Salaffi et al. } \\
\quad(2020)\end{array}$ & 165 & $\mathrm{TC}$ & $\begin{array}{l}\text { A gravidade da TC de tórax está relacionada com } \\
\text { a gravidade e o prognóstico da COVID-19 }\end{array}$ \\
\hline $\begin{array}{l}\text { Changes in lung ultrasound of } \\
\text { symptomatic healthcare professionals } \\
\text { with COVID-19 pneumonia and their } \\
\text { association with clinical findings }\end{array}$ & $\begin{array}{l}\text { Mafort et } \\
\text { al. }(2020)\end{array}$ & 409 & POCUS & $\begin{array}{l}\text { Esse Método desempenha um papel fundamental } \\
\text { na caracterização do envolvimento pulmonar de } \\
\text { pacientes com COVID-19 }\end{array}$ \\
\hline $\begin{array}{c}\text { Spectrum of chest computed } \\
\text { tomographic }(\mathrm{CT}) \text { findings in } \\
\text { coronavirus disease-19 (COVID-19) } \\
\text { patients in India }\end{array}$ & $\begin{array}{l}\text { Parry et al. } \\
\text { (2020) }\end{array}$ & 147 & $\mathrm{TC}$ & $\begin{array}{c}\text { Pacientes com TC positivo apresentaram as } \\
\text { mesmas características de TC relatadas em outras } \\
\text { séries, com predominância de GGOs em } \\
\text { distribuição bilateral e multilobar com predileção } \\
\text { posterior e periférica }\end{array}$ \\
\hline $\begin{array}{l}\text { Point-of-Care Lung Ultrasound for } \\
\text { COVID-19: Findings and Prognostic } \\
\text { Implications From } 105 \text { Consecutive } \\
\text { Patients }\end{array}$ & $\begin{array}{l}\text { Yasukawa } \\
\text { et al. }(2021)\end{array}$ & 105 & POCUS & $\begin{array}{c}\text { A maioria dos pacientes hospitalizados com } \\
\text { COVID-19 tinha anormalidades de ultrassom } \\
\text { pulmonar na admissão e esse método pode ajudar } \\
\text { na estratificação de risco }\end{array}$ \\
\hline
\end{tabular}

Fonte: Autores.

Dentre esses estudos, ao considerar a escala de Newcastle-Ottawa para avaliação da qualidade metodológica, dois tiveram escore 9, três obtiveram escore 8 e 7, conforme demonstrado na Figura 2. 
Figura 2. Avaliação da qualidade.

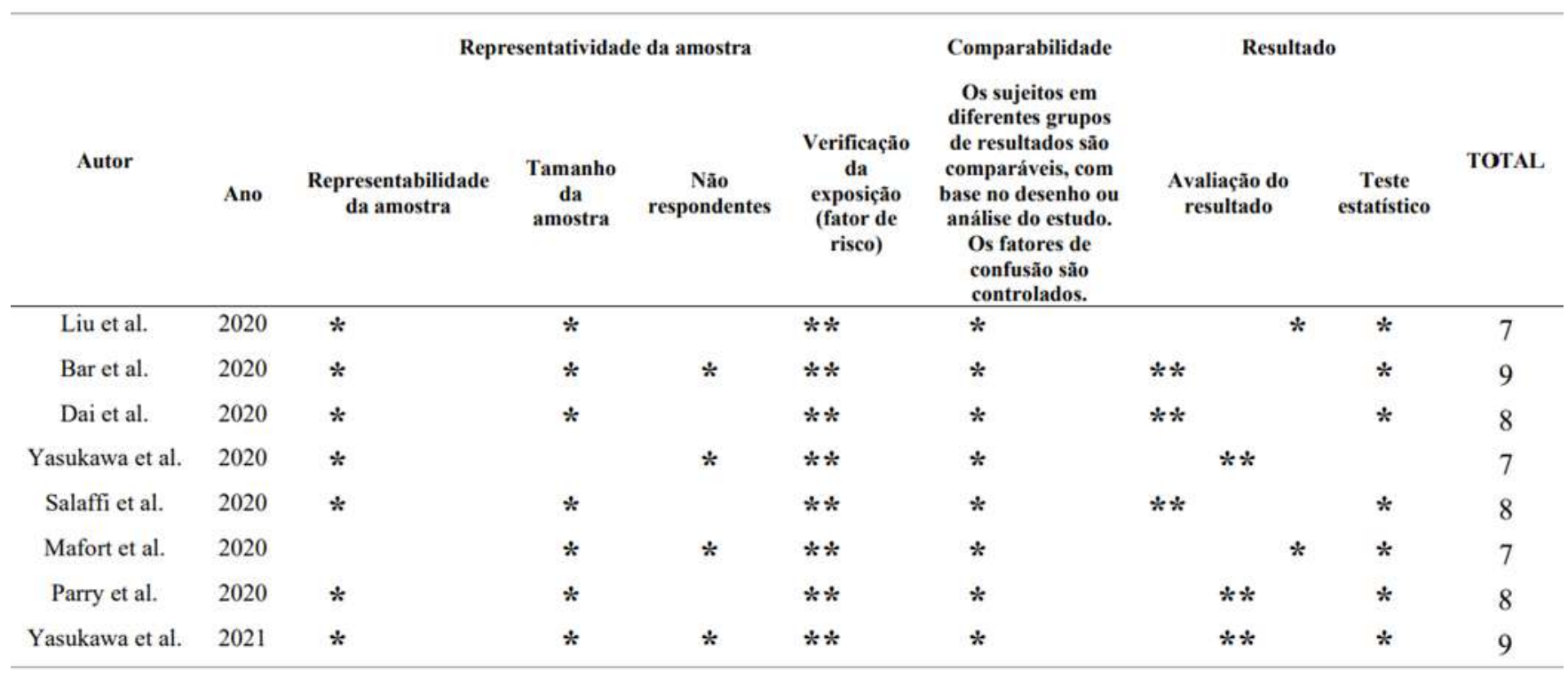

Fonte: Autores.

\section{Discussão}

Os estudos de Dai et al. (2020), Liu et al. (2020), Parry et al. (2020) e Salaffi et al. (2020) utilizaram a Tomografia Computadorizada (TC) para avaliar o nível de comprometimento pulmonar de pacientes, enquanto os estudos de Bar et al. (2020), Mafort et al. (2020), Yasukawa et al. (2020) e Yasukawa et al. (2021) avaliaram através da Ultrassonografia Pulmonar Poin-OfCare (POCUS).

\subsection{Principais achados radiológicos}

Em todos os estudos que utilizaram a TC, a alteração mais evidente foi a opacificação em vidro fosco (ou GGO), definida como o aumento da densidade do pulmão com o não escurecimento das estruturas vasculares e brônquicas, sendo identificado mais precocemente. A consolidação pulmonar é a segunda alteração mais evidente, diferentemente da opacificação em vidro fosco, é presente nas fases mais tardia da infecção (Dai et al., 2020; Liu et al., 2020; Parry et al., 2020; Salaffi et al., 2020).

Na consolidação há o aumento da densidade pulmonar e as estruturas vasculares e brônquicas são escurecidas, devido a troca do ar alveolar por exsudato. Outras alterações menos evidentes, porém, importantes, foram espessamento pleural adjacente, reticulações, dilatação brônquica, espessamento da parede brônquica, espessamento do septo interlobular, nódulos, enfisema, halo pulmonar, halo invertido, aumento de vasos perilesional/intralesional e, mais raramente, derrame pleural, sendo evidente em pacientes mais graves, sugerindo um pior prognóstico. Além disso, os estudos definiam as características desses achados, evidenciando o acometimento de múltiplos lobos pulmonares, distribuídos mais perifericamente e bilateralmente na maioria dos pacientes (Dai et al., 2020; Liu et al., 2020; Parry et al., 2020; Salaffi et al., 2020). 
Figura 3. Achados da TC em pacientes infectados pelo Sars-CoV-2.

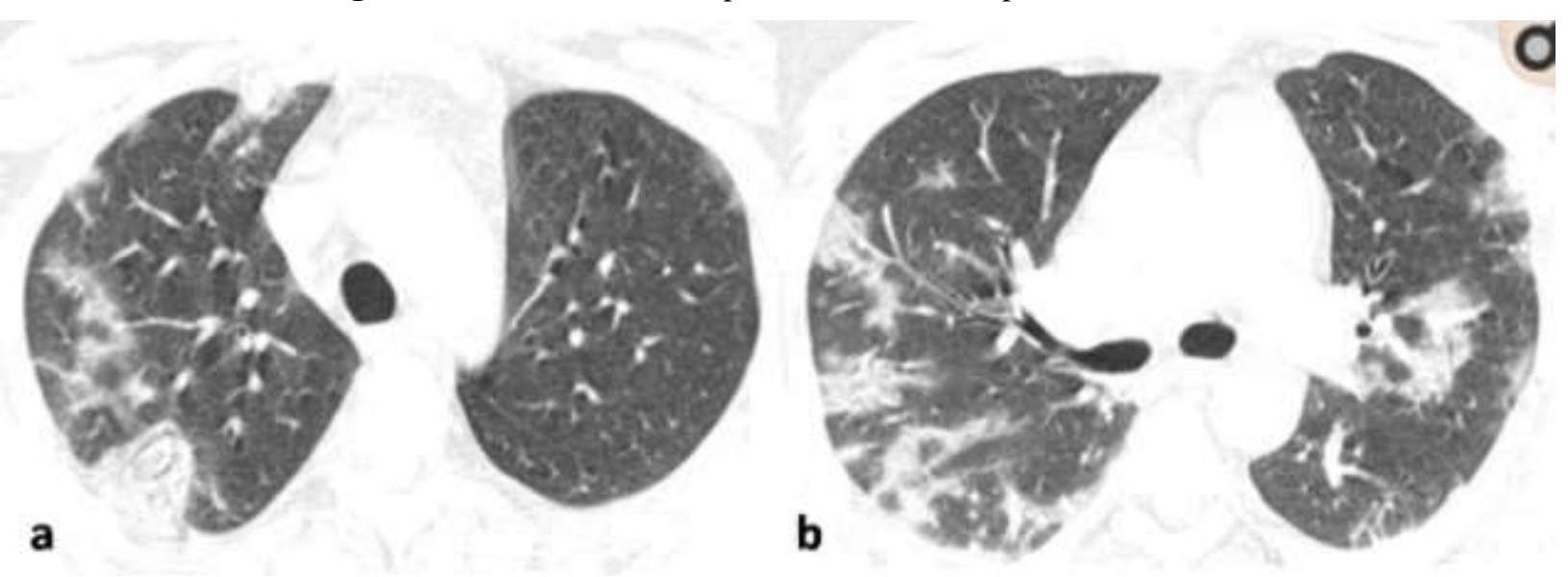

Fonte: Parry et al., 2020 (adaptado).

O estudo de Parry et al. (2020) (figura 3) apresenta imagens de uma TC de tórax em corte axial sem contraste (a e b), sendo visível opacificação em vidro fosco irregulares distribuídos em múltiplos lobos perifericamente (a), com progressão para consolidação (b), visto que a consolidação é mais presentes na fase tardia da doença, esses achados também foram relatados nos estudos de Dai et al. (2020), Liu et al. (2020) e Salaffi et al. (2020).

Em contrapartida, quatro estudos utilizaram a POCUS, esse exame, geralmente é utilizado, em pacientes com suspeita de COVID-19, quando outros tipos de exames de imagem não estão disponíveis. Assim sendo, os achados radiológicos mais frequentes incluíram consolidação irregulares, um dos estudos, identificou foguetes de vidro característicos com ou sem variante Birolleau em todos os pacientes. Os foguetes de vidro e a variante Birolleau indicam áreas de vidro fosco na TC. Soma-se a isso, outros achados: descontinuação, irregularidade e espessamento da linha pleural, Linhas B visíveis sob a pleura e consolidações subpleurais (figura 4) (Bar et al., 2020; Mafort et al., 2020; Yasukawa et al., 2020; Yasukawa et al., 2021)

Figura 4. Principais achados da POCUS.

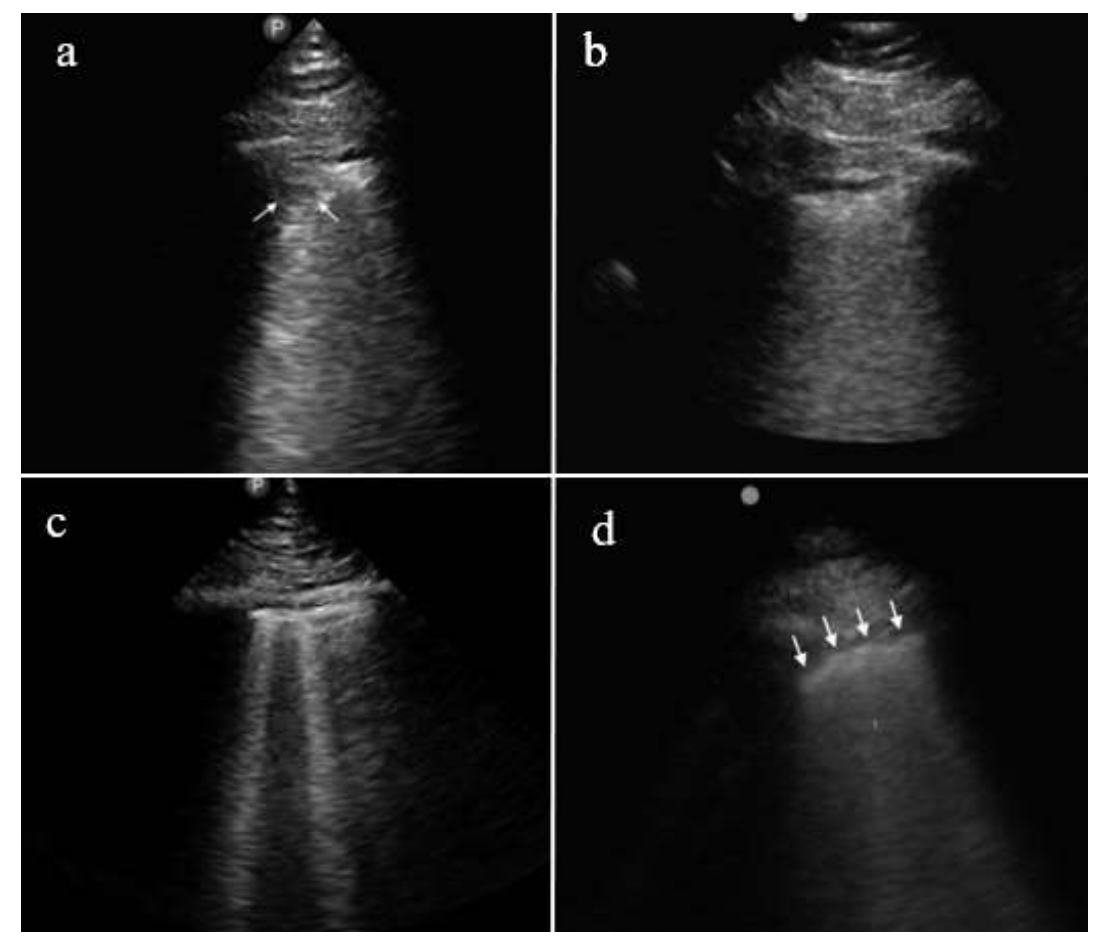

Fonte: Yasukawa et al., 2020 (adaptado). 
A Figura 4 (a, b, c, d) apresenta esses principais achados, adaptados do estudo de Yasukawa et al. (2020) e evidenciados pelos demais estudos que utilizam a POCUS (Bar et al., 2020; Mafort et al., 2020; Yasukawa et al., 2021). Nota-se uma pequena consolidação subpleural (a), a variante Birolleau (pulmão branco) (b), linhas B confluentes (c) e linha pleural espessa e irregular (d). A POCUS pode ser facilmente realizada à beira do leito, além de não expor o paciente à radiação, isso é um dos pontos positivos quando comparada à TC, sendo um exame de imagem sensível para detectar alterações pulmonares.

\subsection{Relação entre os achados, gravidade e fatores de risco}

Nesse contexto, todos os estudos incluídos apresentaram os principais sinais e sintomas dos pacientes, a dispneia, febre, tosse e fadiga foram os mais indicados, esses sintomas estão associados com a gravidade da doença. Desse modo, houve baixa prevalência dos achados radiológicos em pacientes assintomáticos, sendo os pacientes que apresentaram sintomas tiveram maior gravidade e, consequentemente, maior comprometimento pulmonar (Bar et al., 2020; Dai et al., 2020; Liu et al., 2020: Mafort et al., 2020; Parry et al., 2020; Salaffi et al., 2020; Yasukawa et al., 2020; Yasukawa et al., 2021).

Desse modo, sete dos oito estudos avaliaram as comorbidades dos pacientes, sendo as mais evidentes: hipertensão arterial sistêmica, diabetes e outras doenças pulmonares, a prevalência de comorbidades também influenciam na quantidade de achados radiológicos, uma vez que estão diretamente ligados ao aumento da gravidade da doença e, consequente, aumento das sequelas. Alguns estudos relatam que essas lesões pulmonares foram totalmente absorvidas em mais da metade dos pacientes após algumas semanas de alta, indicando que os danos causados pelo Sars-CoV-2 podem ser reparados. Contudo, algumas sequelas residuais podem persistir e que futuramente necessitariam de grande atenção dos serviços de saúde (Dai et al., 2020; Liu et al., 2020: Mafort et al., 2020; Parry et al., 2020; Salaffi et al., 2020; Yasukawa et al., 2020; Yasukawa et al., 2021).

\section{Conclusão}

Os resultados mostram que a infecção provocada pelo Sars-CoV-2 ocasiona diversas alterações no sistema respiratório dos pacientes, sendo possível identificá-las pelos exames de imagens, estando diretamente relacionadas com a apresentação de sintomas e fatores de risco/comorbidades, uma vez que indicam o grau de comprometimento pulmonar, gravidade e prognóstico. É importante frisar, ainda, que esses achados radiológicos não substituem a RT-PCR na confirmação diagnóstica, sendo necessário sempre que possível a confirmação laboratorial para realizar a conduta correta e descartar outras patologias pulmonares. Quanto às limitações, poucas bases de dados foram consultadas e, ainda, há poucos estudos que apresentem as alterações identificadas por outros tipos de exames de imagem. Sendo necessário que estudos futuros abordem outros métodos de diagnóstico por imagem.

\section{Referências}

Bar et al. (2020). The association of lung ultrasound images with COVID-19 infection in an emergency room cohort. Anaesthesia, 75(12), 1620-1625. https://pubmed.ncbi.nlm.nih.gov/32520406/

Chamas, C. (2020). Inovação, propriedade intelectual e acesso a medicamentos e vacinas: o debate internacional na pandemia da Covid-19. Liinc Em Revista, 16(2), e5338. https://doi.org/10.18617/liinc.v16i2.5338

Dai et al. (2020). Temporal changes of CT findings between non-severe and severe cases of COVID-19 pneumonia: a multi-center, retrospective, longitudinal Study. International Journal of Medical Sciences, 17(17), 2653-2662. https://pubmed.ncbi.nlm.nih.gov/33162793/

Dias et al. (2020). Guidelines on the Diagnosis, Treatment and Isolation of Patients with COVID-19. Journal of Infection Control, 9(2), 1-20. http://jicabih.com.br/index.php/jic/article/view/295/pdf_1

Duarte, P. M. (2020). COVID-19: Origem do novo coronavirus. Brazilian Journal of Health Review, 3(2), 3585-3590. https://doi.org/10.34119/bjhrv3n2-187

Galvão et al. (2015). Principais itens para relatar Revisões Sistemáticas e Metanálises: A recomendação PRISMA. Epidemiologia e Serviços de Saúde, 24, 335342. http://scielo.iec.gov.br/scielo.php?script=sci_arttext\&pid=S1679-49742015000200017\&nrm=iso 
Research, Society and Development, v. 10, n. 8, e57010817502, 2021

(CC BY 4.0) | ISSN 2525-3409 | DOI: http://dx.doi.org/10.33448/rsd-v10i8.17502

Gorbalenya et al. (2020). Severe acute respiratory syndrome-related coronavirus: The species and its viruses - a statement of the Coronavirus Study Group. BioRxiv, 2020.02.07.937862. https://doi.org/10.1101/2020.02.07.937862

Higgins et al. (2021). Manual Cochrane para revisões sistemáticas de intervenções | Treinamento Cochrane. Cochrane. https://training.cochrane.org/handbook/current

Lima et al. (2020). Diagnóstico da COVID-19: importância dos testes laboratoriais e dos exames de imagem. Research, Society and Development, 9(9), e259997162. https://doi.org/10.33448/rsd-v9i9.7162

Liu et al. (2020). The pulmonary sequalae in discharged patients with COVID-19: a short-term observational study. Respiratory Research, 21(1), 125. https://pubmed.ncbi.nlm.nih.gov/32448391/

Machado et al. (2021). Pandemias e COVID-19 transformam o mundo: uma análise de contextos. Enfermagem Brasil. 10.33233/eb.v20i2.4442. 20(2), 255-279. https://doi.org/10.33233/eb.v20i2.4442

Mafort et al. (2020). Changes in lung ultrasound of symptomatic healthcare professionals with COVID-19 pneumonia and their association with clinical findings. Journal of Clinical Ultrasound: JCU, 48(9), 515-521. https://pubmed.ncbi.nlm.nih.gov/32827163/

Mogami et al. (2021). Chest computed tomography in covid-19 pneumonia: A retrospective study of 155 patients at a university hospital in Rio de Janeiro, Brazil. Radiologia Brasileira, 54(1), 1-8. https://doi.org/10.1590/0100-3984.2020.0133

Ouzzani et al. (2016). Rayyan—a web and mobile app for systematic reviews. Systematic Reviews, 5. https://doi.org/10.1186/s13643-016-0384-4

Page et al. (2021). The PRISMA 2020 statement: An updated guideline for reporting systematic reviews. In The BMJ (Vol. 372). BMJ Publishing Group. https://doi.org/10.1136/bmj.n71

Parry et al. (2020). Spectrum of chest computed tomographic (CT) findings in coronavirus disease-19 (COVID-19) patients in India. European Journal of Radiology, 129, 109147. https://pubmed.ncbi.nlm.nih.gov/32623113/

Rosa et al. (2020). COVID-19 findings identified in chest computed tomography: a pictorial essay. In Einstein (Sao Paulo, Brazil) (Vol. 18, p. eRW5741). NLM (Medline). https://doi.org/10.31744/einstein_journal/2020RW5741

Salaffi. (2020). The role of a chest computed tomography severity score in coronavirus disease 2019 pneumonia. Medicine, 99(42), e22433. https://pubmed.ncbi.nlm.nih.gov/33080676/

Wells et al. (2021). The Newcastle-Ottawa Scale (NOS) for assessing the quality of nonrandomised studies in meta-analyses. Ottawa Hospital Research Institute. http://www.ohri.ca/programs/clinical_epidemiology/oxford.asp

Woo et al. (2012). Discovery of Seven Novel Mammalian and Avian Coronaviruses in the Genus Deltacoronavirus Supports Bat Coronaviruses as the Gene Source of Alphacoronavirus and Betacoronavirus and Avian Coronaviruses as the Gene Source of Gammacoronavirus and Deltacoronavirus. Journal of Virology, 86(7), 3995-4008. https://doi.org/10.1128/jvi.06540-11

Yasukawa et al. (2020). Point-of-Care Lung Ultrasound Findings in Patients with COVID-19 Pneumonia. The American Journal of Tropical Medicine and Hygiene, 102(6), 1198-1202. https://pubmed.ncbi.nlm.nih.gov/32333544/

Yasukawa et al. (2021). Point-of-Care Lung Ultrasound for COVID-19: Findings and Prognostic Implications From 105 Consecutive Patients. Journal of Intensive Care Medicine, 36(3), 334-342. https://pubmed.ncbi.nlm.nih.gov/33535883/

Zhu et al. (2020). A Novel Coronavirus from Patients with Pneumonia in China, 2019. New England Journal of Medicine, $382(8)$, 727-733. https://doi.org/10.1056/nejmoa2001017 